\title{
Beyond Conventional Event-related Brain Potential (ERP): Exploring the Time-course of Visual Emotion Processing Using Topographic and Principal Component Analyses
}

\author{
Gilles Pourtois · Sylvain Delplanque • \\ Christoph Michel · Patrik Vuilleumier
}

Accepted: 4 February 2008/Published online: 13 March 2008

(C) Springer Science+Business Media, LLC 2008

\begin{abstract}
Recent technological advances with the scalp EEG methodology allow researchers to record electric fields generated in the human brain using a large number of electrodes or sensors (e.g. 64-256) distributed over the head surface (multi-channel recording). As a consequence, such high-density ERP mapping yields fairly dense ERP data sets that are often hard to analyze comprehensively or to relate straightforwardly to specific cognitive or emotional processes, because of the richness of the recorded signal in both the temporal (millisecond time-resolution) and spatial (multidimensional topographic information) domains. Principal component analyses (PCA) and topographic analyses (combined with distributed source localization algorithms) have been developed and successfully used to deal with this complexity, now offering powerful alternative strategies for data-driven analyses in complement to more traditional ERP analyses based on waveforms and peak measures. In this paper, we first briefly review the basic principles of these approaches, and then describe recent ERP studies that illustrate how they can inform about the precise spatio-temporal dynamic of emotion processing. These studies show that the perception of emotional visual stimuli may produce both quantitative
\end{abstract}

G. Pourtois · P. Vuilleumier

Laboratory for Behavioral Neurology \& Imaging of Cognition,

Department of Neuroscience \& Clinic of Neurology, University

of Geneva, Geneva, Switzerland

G. Pourtois $(\varangle) \cdot$ S. Delplanque $\cdot$ P. Vuilleumier

Swiss Center for Affective Sciences, University of Geneva,

7 rue des Battoirs, 1205 Geneva, Switzerland

e-mail: gilles.pourtois@medecine.unige.ch

C. Michel

Functional Brain Mapping Laboratory, Department of

Neurology, University Hospital, Geneva, Switzerland and qualitative changes in the electric field configuration recorded at the scalp level, which are not apparent when using conventional ERP analyses. Additional information gained from these approaches include the identification of a sequence of successive processing stages that may not fully be reflected in ERP waveforms only, and the segregation of multiple or partly overlapping neural events that may be blended within a single ERP waveform. These findings highlight the added value of such alternative analyses when exploring the electrophysiological manifestations of complex and distributed mental functions, as for instance during emotion processing.

Keywords Scalp EEG · High-density ERP mapping · Temporal PCA - Spatial PCA · Topography · Field strength · Dissimilarity · Clustering - Emotion perception . Vision - Early perceptual effect $\cdot$ P3a $\cdot$ P3b.

\section{Limits of Conventional ERP Data Analysis}

Given its millisecond time-resolution and direct relationship to neuronal activity (i.e., post-synaptic dendritic potentials of a large number of neurons activated synchronously and arranged in a geometrical configuration such as to yield a dipolar field), scalp electro-encephalogram (EEG) is a highly valuable time-resolved brain-imaging technique (see $[1,2]$ ). Event-related brain potentials (ERPs) are computed from the EEG by using, in the vast majority of cases, the averaging of data as a signal extraction technique (see $[3,4]$ for different techniques, including frequency and single trials analyses). EEG epochs are time-locked to the same event class (either a stimulus or a response), and then averaged to yield a waveform carrying a mean amplitude value at each time-point, whose successive negative and 
positive deflections over time are thought to reflect specific stages of sensory, cognitive, or decision-related processes [5].

According to published guidelines ([6], p. 141), "the simplest approach is to consider the ERP waveform as a set of waves, to pick the peaks (and troughs) of these waves, and to measure the amplitude and latency at these deflections". These peak amplitude measurements are not representing absolute values of electric brain activity, but are obtained either relative to a pre-stimulus baseline (baseline to peak analysis) or sometimes to an immediately preceding or following peak (peak-to-peak analysis). "Relevant" electrophysiological events are therefore selected a priori by searching for electrodes with potential peaks that can be either negative or positive deflections depending on the actual configuration of the underlying generators. Although this "simple" ERP analysis method has proven its immense powerfulness to shed light on the time-course of various cognitive and emotional processes in the human brain (see [5, 7] for recent reviews), the experimenter using a conventional ERP technique has to adhere to a number of prerequisites and be aware of some of the limitations bound to this specific data analysis.

Among them, a key assumption underlying the ERP analysis method is that potentially interesting aspects of cortical brain processes are primarily reflected in these maxima (peaks) but, by extension, not discernible when the amplitude is low or close to zero [6]. However, this conjecture is not verified by neurophysiological data as low EEG/ERP signal amplitude does not mean absence of important neuronal events [2,3]. In addition, difficulties may arise because the latency of a peak may vary somewhat across different electrodes, a limitation that becomes more obvious when increasing the number of channels. This concern has led some researchers to identify peaks using a measurement of Global Field Power (GFP), which is defined as the spatial root mean squared across all electrodes and which is reference-independent ([8], see Fig. 2 of [9]). GFP has the clear advantage of providing a global and spatially unbiased measure of the electric field strength at the scalp, which is related to the amount of synchronously active neurons in the brain [10]. The GFP measure is therefore a general estimate of the electric signal amplitude at each time point despite slight variations of individual peak latencies across different electrode positions [11].

Another problem associated with the conventional ERP method concerns the location of the reference electrode (the so-called "reference-problem" in ERP literature; see [11, 12], see Fig. 1 of [9]). Waveform analyses (and amplitude measurements of peaks thereof) are heavily influenced by the reference. The amplitude of a component's peak identified at one electrode location can radically change (and sometimes even cross the zero baseline and switch polarity) as a function of the position of the reference electrode. Changing the reference also changes statistical outcomes. By contrast, analysis methods that consider the spatial distribution of the ERP, such as microstate segmentation [8, 13] and (spatial) PCA [14], are reference-independent. This is because the configuration of the scalp topography is independent of the specific reference electrode [13, 15]. When calculating the voltage distribution (using interpolation methods such as spherical splines; see [16]), the resulting equipotential lines (reflecting subtle borders and changes in the distribution of the electric field over the scalp surface) remain exactly the same, and unlike conventional ERPs, the electric "landscape" remains unaffected by changes in the recording montage (see [11] for a recent demonstration). For technically oriented considerations related to the inverse solution problem itself (e.g., the violation of the quasistationary state assumption), the average reference of the surface potential is usually calculated and used for subsequent data analysis looking at the spatial distribution of the ERPs [8, 9, 11, 17].

Hence, to circumvent some of the difficulties associated with the conventional ERP analysis method [6] but also to deal more effectively with the increasing complexity of the current ERP data sets nowadays routinely obtained with multiple channels [18], modern data-driven analyses (such as microstate segmentation and PCA) have been developed and used to study the spatio-temporal dynamics of various domains of human cognition [19-21] and emotion [22, 23]. Microstate segmentation is also sometimes called topographic pattern analysis [9]. In both cases, it refers to a whole set of ERP data analyses (allowing to test for and tease apart differences in strength, topography, latency and component sequence), as we introduce and illustrate in the next sections.

Importantly, microstate segmentation and PCA provide the clear advantage of minimizing the amount of userdependent biases and a priories (e.g., assuming that relevant aspects of cognitive or emotional processes would mainly be reflected in peaks, see [6]). Both microstate segmentation and PCA can give new insights on the timecourse and structure of brain activity associated with specific cognitive or emotional events, without the need to restrict a priori the ERP analysis to a few time points only (e.g., where the amplitude is visibly high) and/or to a few electrode positions only [14, 19], as in conventional ERP analysis [6].

Below, we will first shortly present the basic principles of the microstate segmentation $[8,9,19,24,25]$, whose primary aims are to identify dominant topographic scalp maps in multi-channel ERP data sets, and to compare the strength and expression of these maps over time and across 
experimental conditions. To illustrate the value of this approach, we will then present a recent study using ERP segmentation to investigate the neural mechanisms of attentional capture by emotional (threat-related) stimuli $[26,27]$. In the second section of this review, we will then briefly present the rationale of temporal (and spatial) PCA [14] before turning to concrete empirical studies that illustrate how PCA can inform about the precise neurophysiological dynamics of visual emotion processing [2830]. Specifically, we will focus on the dissociation between the effects of valence and arousal, which correspond to intrinsically mingled components of emotion (see [29]).

\section{General Principles of Microstate Segmentation}

The general statistical principles of the microstate segmentation have been described extensively elsewhere $[9,11,13,19,20,31]$. Therefore, here we only provide a brief overview of this approach to ERP data analysis, before presenting an application in the context of emotional attention [23, 32]. Note that all steps of analysis described here can be performed using a dedicated software, CARTOOL, developed by Denis Brunet (http:// brainmapping.unige.ch/Cartool.htm). The rationale of microstate segmentation is to objectively summarize the complex topographic information embedded in high-density ERPs, and to offer a high degree of interpretational power with regards to the nature and extent of putative electrophysiological differences between experimental conditions (strength, topography, latency shift or component sequence, see [9] for a thorough presentation and discussion).

When interpolating local amplitude values recorded at each channel to topographic voltage maps (e.g., for instance with spherical splines, see [16]) and then inspecting the succession of these topographic maps following stimulus or response onset, a highly reproducible observation is that evoked activities appear to remain stable for several tens of milliseconds, before a more or less sharp qualitative change in the electric field configuration may occur and lead to another topographic map, which may in turn remain stable for a certain duration (a phenomenon originally referred to as "functional microstates", see [8, 13, 19, 33, 34]). The rationale of the microstate segmentation is to isolate these periods of temporal stability (and by extension changes) in the manner that the global electric field is distributed over the scalp surface and over time points, by using a formal statistical approach applied to the whole topography information rather than to values from single electrodes [11]. This stems from the fact that different map topographies reflect different configurations of electric sources in the brain, that is, different neural networks [35-37]. As a caveat, it is important to note that the symmetrical statement is not true: different sources in the brain do not necessarily translate as different topographies on the scalp $[8,25]$.

A straightforward way to compare scalp map topographies was already proposed some time ago in the pioneer neurophysiological work of Lehmann and Skrandies [8] and involves calculating a Global Dissimilarity index across successive maps. Global dissimilarity is obtained by computing the square root of the mean of the squared differences between all corresponding electrodes, once these maps have been recalculated against the average reference and normalized to unitary strength (i.e., divided by its own GFP, see [8], see Appendix 1 of [9]). Global dissimilarity is inversely related to the spatial correlation between two maps (i.e., low global dissimilarity values indicate similar topographies, while high global dissimilarity values indicate topography changes). Using this measure of topographic similarity-dissimilarity, it is possible to compute the stability of successive maps over time (and thus identify functional microstates), as well as to statistically compare the different scalp topographies between experimental conditions [9]. Differences in global dissimilarity are evidenced using non-parametric bootstrapping procedures (including Monte Carlo MANOVA, see $[27,31,38,39]$ cognitive applications); an analysis colloquially referred to as TANOVA (topographic ANOVA). Importantly, using global dissimilarity, it may be possible to reveal topographic changes over the scalp that do not necessarily coincide in time with any reliable changes in the global strength of the signal, or that may arise during time-periods where the ERP amplitude is actually low or close to baseline. Thus, the occurrence of important topographic changes at time-points with low (local or global) amplitude in ERP signals clearly refutes the classic assumption that only peaks (or maxima) are relevant electrophysiological phenomena (as hypothesized by the conventional analysis).

By combining the statistical comparison of topographies using global dissimilarity with the comparison of field strength using the Global Field Power, unequivocal conclusions about the nature of the electrophysiological differences can be made. Thus, these measures can inform about the actual electrophysiological correlates of specific cognitive or emotional processes in the brain, and tease apart genuine amplitude/strength effects from qualitative changes in the configuration of intracranial generators $[8$, $9,13,36]$. It has to be emphasized that the conventional ERP peak analysis cannot distinguish topographic from strength differences. Amplitude differences at certain electrodes may be due to differences in the configuration of the electric field as well as differences in strength of the same topography. 
Global dissimilarity is useful to inform about time points where reliable change in the distribution of the electric field may occur following either the stimulus or the response onset (or any other time segment of interest). However, this analysis does not allow defining unique map configurations and does not formally test whether a specific map configuration is suppressed (or conversely whether it is prolonged or delayed) in one condition (or time-period) as compared with another condition (or time-period). For instance, the global dissimilarity measure alone is not sufficient to determine whether topographic differences are explained by a single or multiple configuration transformation, by a simple latency shift, or by a sequence change for a given topography across conditions. But clear answers to these important questions can be obtained using a pattern analysis of the ERP scalp topographies [25]. In brief, pattern analysis can efficiently summarize ERP data by a limited number of distinctive field configurations (so-called microstates, [13]). The spatio-temporal segmentation algorithm is derived from a k-mean spatial cluster analysis [25] and identifies the most dominant scalp topographies appearing in the group-averaged ERPs of each condition and over time. K-mean is a classical and general clustering algorithm. The optimal number of topographic maps explaining the whole data set is determined objectively for example by cross validation [25]. Cross validation criterion was first introduced by Pascual-Marqui et al. [25] as a modified version of the predictive residual variance (see Appendix 1 in [9] for details and mathematical equations). Its absolute minimum gives the optimal number of segments. Here we focus on the k-mean clustering used in conjunction with cross validation because a large number of high-density ERP mapping studies to date have applied this method [11]. However, it must be noted that there are newer alternative clustering algorithms, such as the agglomerative hierarchical clustering that has been specifically designed for the analysis of EEG/ERPs (see [9] for a discussion and comparison of different clustering methods; see [40] a recent cognitive application).

The dominant scalp topographies (identified in the group-averaged data) are then fitted to the ERPs of each individual subject using spatial fitting procedures to quantitatively determine their representation across subjects and conditions. This procedure thus provides finegrained quantitative values, such as the duration of the map (including its precise onset and offset times), the global explained variance (or goodness of fit), and the strength of the map, which are critical indices of the significance of a given scalp topography [19]. This information is not available otherwise in a classical component analysis. Parametric statistical tests can then be performed on these variables (duration, goodness of fit, or strength) in order to compare different experimental conditions or time-periods, and eventually disclose the electrophysiological correlates associated with one specific condition or time-period.

Once the complex ERP topographic time-series is reduced to a smaller number of dominant scalp maps using this pattern analysis, a final (but optional) step in the pattern analysis may apply a source localization algorithm to estimate the location of intracranial generators at the origin of the dominant maps recorded on the scalp surface. Alternatively, sources estimation can also be performed directly using the single-subject data over the time-period when a given map predominates and/or when topographies significantly differ and/or when the GFP differs. A wide range of source localization algorithms have been proposed to address the source localization problem (see [11, 41] for reviews). A common method involves distributed linear inverse solutions. This class of inverse models is based on a reconstruction of the brain electric activity at each point of a 3D grid of solution points (i.e., much larger than the number of measurement points on the surface), wherein each point represents the center of gravity of a local current dipole with a certain strength and orientation [11]. Because the problem is highly underdetermined, a priori constraints are needed to derive a unique solution. Such constraints are based on minimizing the overall intensity [42], maximizing the smoothness (LORETA, [43]), or integrating biophysical laws about the regression of electric activity in space (LAURA, [44]). Unlike dipole solutions [45], distributed inverse solutions compute multiple simultaneously active sources without any a priori assumption on the number and position of the underlying cortical generators (see [27, 31] for emotion and cognitive applications, respectively). Reliable differences in the active sources between topographic maps (or time-periods) can be evidenced using parametric or non-parametric statistical tests. It is important to emphasize that any source localization applied to these segmentation maps is thus based on the statistical evidence that the electric fields were different [8, 11], a requirement which is not always met when using a conventional ERPs analysis.

\section{Using Topography Segmentation to Study Attentional Capture by Threat Cues}

In a recent ERP study [26], we used a modified version of the classic dot-probe task [46, 47] in normal (non-anxious) adult participants while we recorded high-density EEG to track the time-course of spatial orienting toward the location of emotional stimuli. Cues were faces with fearful or neutral expression, appearing briefly prior to a single neutral target (a white bar) presented at the same location as one of the faces. On each trial, two faces were first shown together, for a duration of $100 \mathrm{~ms}$, one in the left 
visual field (LVF) and one in the right visual field (RVF), one neutral and one with a fearful expression. The faces were then replaced by a small bar-probe (duration of $150 \mathrm{~ms}$ ), oriented either vertically or horizontally, appearing at the position previously occupied by one of the faces. All stimuli (faces and bar-probe) were presented in the upper visual field to allow us to measure early retinotopic responses in ERPs [48]. Participants were asked to perform a go/no-go matching task in which they had to judge, on each trial, whether the orientation of the barprobe (in the LVR or RVF) matched that of the thicker line-segment within the fixation cross (see [23, 26] for methodological details). Only ERPs for no-go trials (ruling out any confounding motor-related activity) were analyzed. The bar-probe could appear either on the side of the fearful face (valid condition) or on the side of the neutral face (invalid condition), in an unpredictable (50\% each) and randomized manner. However, faces were entirely irrelevant to the participants' task. Moreover, since participants had to fixate the central cross, emotional cues appeared at unattended locations, allowing us to assess any reflexive biases in the spatial distribution of attention to peripherally presented probes. We used only short-time intervals between the face pair and the bar onset (100-300 ms, systematically randomized) to tap exogenous mechanisms of spatial orienting [49].

Our main question was whether sensory responses to the peripheral bar-probes would be enhanced when replacing a fearful (valid) face, rather than a neutral (invalid) face, as predicted if spatial attention was involuntarily oriented toward that particular location (emotional attention, see $[23,32])$. Our main comparison therefore concerned the amplitude (and latency) of ERP generated by the exact same bar-probe as a function of the different emotional values of the preceding face context.

Conventional analyses [6] on the exogenous visual ERPs confirmed that fearful faces (relative to neutral faces) significantly modulated the early sensory processing of barprobes appearing at the same location. The lateral occipital P1 component peaking at $135 \mathrm{~ms}$ post-stimulus onset was significantly enhanced when the target-bar replaced a valid fearful face as compared with an invalid neutral face (Fig. 1a), even though the bars were always physically identical but differed only due to the preceding emotional face. Source estimation methods based on the LORETA constraint [43] further confirmed that the P1 component was generated in the extrastriate visual cortex, including the middle occipital gyrus and inferior temporal gyrus [26]. Noteworthy, the effect of fearful faces on ERPs to subsequent target-bars was selective for the lateral occipital P1 component, but did not affect other exogenous visual components, such as the earlier $\mathrm{C} 1$ component arising from the primary visual cortex (see [48]) or the subsequent N1 component presumably generated by higher extrastriate areas within occipito-parietal cortex [50].

These results therefore suggest an amplification of sensory responses to a neutral visual stimulus (bar-probes) taking place at early processing stages within extrastriate visual cortex, induced by the preceding fearful face at the same location. This effect is consistent with a gain control mechanism of spatial attention [51], which is thought to enhance visual processing via top-down signals from fronto-parietal areas. The gain control mechanism operates by amplifying the signal-to-noise ratio for attended stimuli in extrastriate visual cortex while suppressing the interfering signal generated by unattended stimuli. In this model, top-down signals are therefore thought to be activated prior to target onset in the case of preparatory/ endogenous attention, or at an early latency post-stimulus onset in the case of reflexive/exogenous attention, so as to enhance the ongoing neural responses in extrastriate cortex [52]. However, using a conventional ERP analysis [26], we did not find any reliable evidence for a differential effect that could precede the $\mathrm{P} 1$, which would potentially originate in fronto-parietal areas responsible for the control of spatial attention and somehow induce the subsequent increase observed at the P1 level.

To better capture these precise spatio-temporal dynamics thought to reflect a gain control mechanism following target onset, we therefore turned to topographical segmentation methods $[8,19]$ that allowed the identification of subtle changes in the topographic configuration of scalp EEG over time, arising despite the absence of any reliable local modulation (at a few electrode positions) or global modulation (GFP) of ERP amplitude. More precisely, we tested whether any differential neural activity (e.g., within the fronto-parietal network) might precede the amplification of P1 responses to bar probes by emotional cues, and thus correspond to the causal source of attentional biases in spatial attention [27].

Firstly, this new analyses indicated that EEG activity during the time range of the P1 component evoked by bar-probes (120-160 ms) did not exhibit any differences in topographic configuration across the different conditions of face cues. There was only a significant increase in the strength of the topography map corresponding to the P1 (as indicated by a higher GFP), when targets followed a valid fearful face as compared with an invalid fearful face (Fig. 1b). But the topography map itself did not differ between conditions, supporting a genuine amplification or "gain mechanism" acting on the same neural network in extrastriate cortex, rather than activation of a different network.

Secondly, and more critically, this topographic analysis revealed the existence of an early ( $<100 \mathrm{~ms}$ post bar-probe onset) and stable (40-80 ms) topographical map that reliably distinguished valid from invalid targets, and arose just 

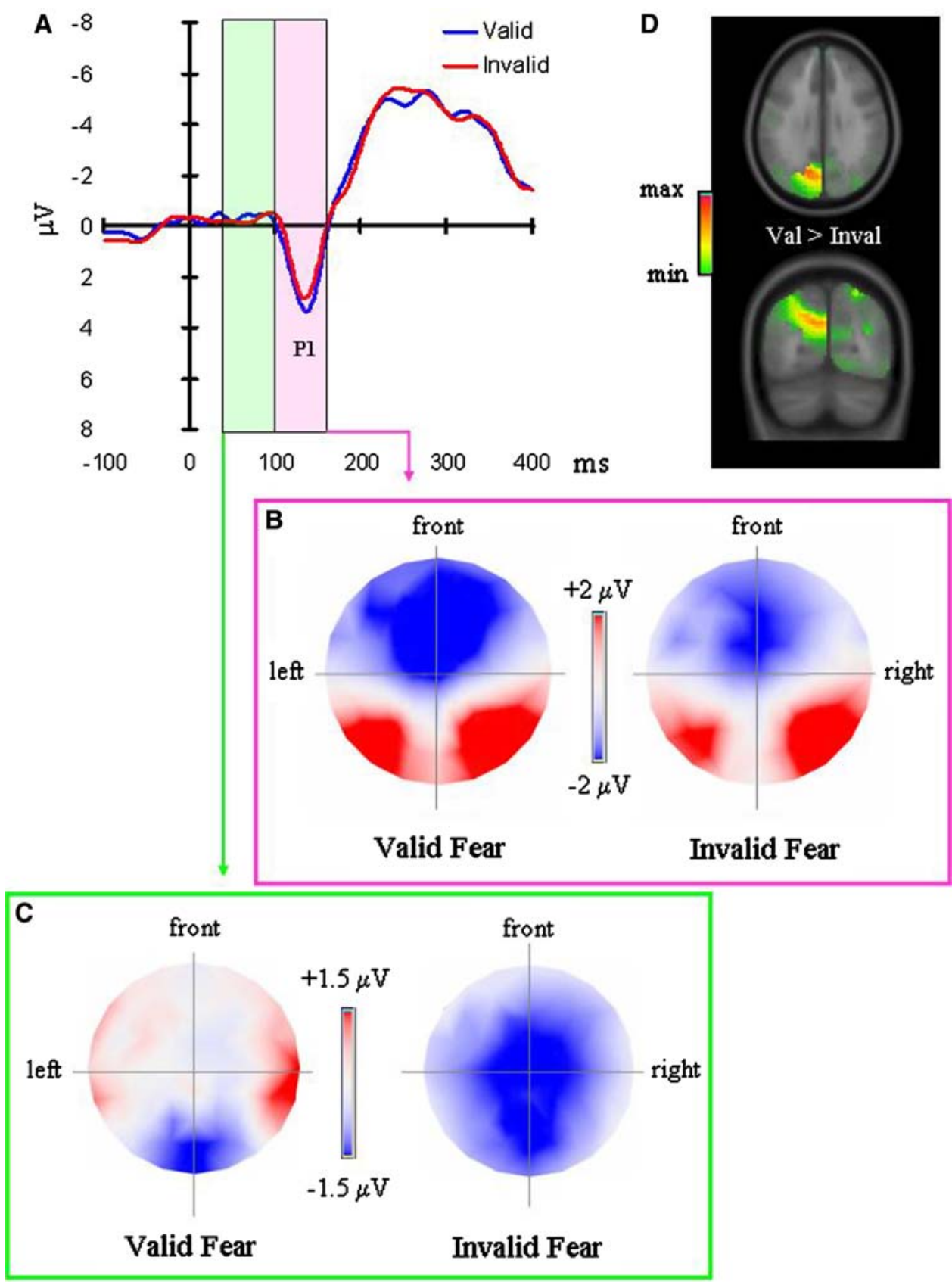

Fig. 1 Illustration of segmentation analysis in a dot-probe task with emotional face cues. (a) Grand average waveforms in the fear valid (blue waveform) and invalid (red waveform) condition (from electrode PO8). The black vertical line bar indicates the onset of the bar probe (target). The P1 waveform (interval highlighted by a pink shaded area) was larger for fear valid as compared with fear invalid trials, although the target stimulus was the same in these two conditions [26]. In the time-window preceding the P1 ERP component, the signal was actually close to zero baseline at occipital electrodes in both conditions (interval highlighted by a green shaded area), although a significant difference in scalp topography was observed between the two conditions. (b) Voltage maps for the P1 in the fear valid and fear invalid conditions (in the 130-140 ms interval

prior to the topographical maps corresponding to P1 (Fig. 1c). Crucially, this topographic modulation was evidenced during a sustained time-period (40-80 ms) shortly following bar-probe onset) showing a more prominent P1 scalp topography in the former than the latter condition but without any qualitative change in the dipolar configuration of this map across conditions (amplitude modulation only). (c) Voltage maps in the fear valid and fear invalid conditions in the 40-80 ms interval following barprobe onset, showing a significant modulation of the global scalp configuration (with no change in amplitude). (d) Statistical parametric mapping provided by LAURA indicated that brain regions that were more activated by fear valid than fear invalid trials in the $40-80 \mathrm{~ms}$ post barprobe onset were mainly located in the left posterior parietal cortex, in a region close to the intraparietal sulcus $(p<0.001$, uncorrected; see [27])

after target onset, when the ERP signal was low and even close to the baseline level (preceding the $\mathrm{C} 1$ and $\mathrm{P} 1 / \mathrm{N} 1$ waveforms elicited by targets, see Fig. 1a), therefore 
making it difficult to be captured by a conventional ERP analysis. The neural sources of this distinctive map could then be estimated by the LAURA algorithm and was found to be clearly different from extrastriate occipital sources associated with the subsequent P1 (120-160 ms). Instead, this early map involved cortical generators in posterior temporal and posterior parietal regions (Fig. 1d). The selective activation in the two latter regions could potentially reflect initial top-down signals and guide subsequent sensory processing in extrastriate visual cortex [52], consistent with the predictions of a gain control mechanism of spatial attention [51].

In summary, our topographic ERP analysis revealed that an early microstate (at $40-80 \mathrm{~ms}$ post-target onset) was significantly more present in the valid condition, when targets appeared at the same versus different location as a fearful face; and that this distinctive configuration of neural activity preceded another microstate (at $120-160 \mathrm{~ms}$ ) corresponding to the $\mathrm{P} 1$, whose generators did not differ across conditions but whose amplitude was enhanced for valid vs. invalid targets (Fig. 1; see [27]). These ERP results are consistent with the idea that a first sweep of activity in posterior temporal and parietal regions might take place rapidly after a visual target onset and possibly provide the signal for subsequent top-down control of target processing $[52,53]$. Here, top-down signals from posterior parietal regions were modulated by emotional significance of the preceding face cue. In further support of this idea, we also found that these two consecutive neural events were positively correlated (using Pearson correlation coefficient, see Fig. 7 in [27]), suggesting a direct functional coupling between the early posterior parietal activity $(40-80 \mathrm{~ms})$ and the subsequent P1 activity (120-160 ms). Although speculative, this enhanced coupling between parietal and extrastriate activity might provide a plausible neural mechanism underlying the facilitation in orienting spatial attention toward targets appearing at the location of threatrelated cues (emotional attention, see [32]).

Microstate segmentation can usefully complement more conventional ERP analyses in a variety of other experimental situations where differences between conditions may involve the addition of an extra processing stage or the modulation of the duration of a specific neural process [5456]. Because emotional processing is typically associated with a complex sequence of stages from appraisal mechanisms to adaptive changes in cognitive systems and feeling states [57], which are likely to unfold along both parallel and serial pathways throughout widespread neural networks, we believe that segmentation and pattern analyses can offer a powerful approach to dissect the temporal dynamics of affective processes in the human brain and their impact on other cognitive operations. Some limitations of this segmentation method might however arise when multiple processing stages overlap with each other in time (see [4]), which would then lead to more variable configurations of electrical topography at the scalp due to the combination of different, simultaneously active microstates. However, the instantaneous summation of electric fields would result in a distinct scalp map corresponding to the linear sum of these different intracranial electric fields at each instant in time. If these intracranial electric fields would vary over time, then the topography should also vary over time, a distinctive spatio-temporal dynamic that should be easily captured in principle by a topographic pattern analysis. In many cases, such temporal overlap might also be simply disentangled by separating common and dissimilar neural generators using a distributed source localization analysis. In other cases, however, overlapping processes might be better distinguished by other approaches, such as alternative source models focusing on current changes at the local scale [58]. Another powerful data-driven method to separate different though temporally overlapping ERP components is provided by PCA, as reviewed in the next section.

\section{General Principles of PCA}

Another family of data-driven analyses for high-density (multi-channel) ERPs is provided by PCA, which shares some similarities with the microstate segmentation although we do not intend to directly compare these two methods in depth in this review article. Different assumptions are required by these two analysis methods (e.g., orthogonality of brain processes with a Varimax rotated PCA) and therefore, one method may be more informative than the other depending on the specific hypotheses and experimental context. Future work should more directly assess the similarities and differences between these two analysis methods (microstate segmentation versus spatial PCA) when they are applied on the exact same high-density ERP data set. It is interesting to note that similar concerns about the definition of a component (as applied by the conventional ERP analysis, see [6]) were raised in the PCA literature as well (see [14]). The underlying criticism was that a peak at a specific latency is not a conservative definition for a component of the ERPs, but that the association to a given cognitive or psychological function should be taken into account as well. This can formerly be assessed by measuring changes related to experimental variables of interest, rather than a priori selecting and focussing on peaks only. Using PCA, it is therefore assumed that a component corresponds to a temporal pattern of activity in a particular brain region (or set of brain regions) that directly relates to a particular cognitive or emotional process $[14,59]$. 
In the context of multi-channel ERP mapping, the goal of PCA is to extract ERP components whose variance is related to the experimental variables [60]. Basically, the PCA can be viewed as a particular case of a broader collection of analysis techniques called factorial analyses. Like microstate segmentation, PCA is essentially an exploratory and descriptive method for summarizing complex, multi-channel ERP data sets by reducing their temporal and/or spatial dimensions. Thus, PCA can provide useful insight into how ERPs components are affected by the experimental manipulations (see [61, 62] for description of the PCA analysis; see [63-67] for additional technical details). Without any a priori assumptions about the shape or number of components in the data set, the PCA will determine the complex relationships between a large number of dependent variables (i.e., the voltage at each time frame for a temporal PCA and the voltage at each electrode for a spatial PCA) and summarize these relations in terms of unobserved dependent variables (what is usually called temporal or spatial factor in a PCA), corresponding to the recorded components. Thus, PCA provides a measure of the contribution of each factor to the observed ERPs, and allows subsequent tests to determine any statistical difference between conditions. The application of PCA is not limited to ERPs and can also concern raw EEG epochs (e.g., [68]), serve as a method to efficiently filter the data (e.g., ocular artefact; [69]), or be used for specific source localization purposes [66, 67].

In this review, we will concentrate on temporal PCA (tPCA) but a similar underlying logic holds for spatial PCA (see [14, 59, 66, 70] for additional information). The first step of the tPCA is to compute the covariances (or correlations) between all pairs of time points over all waveforms. The idea is that time points covarying with each other belong to the same factor. In other words, for time periods that seem to behave similarly across the participants, the conditions and the electrodes are summarized in a common factor. To identify components, the PCA therefore looks for time points that are reliably correlated, instead of arbitrarily focussing on just peaks or valleys expressed in the waveform (unlike conventional ERP analysis).

It is important to emphasize that these new factors represent weighted linear combinations of the original data, a requirement in agreement with the Helmholtz's principle of superposition, thus respecting the electrophysiological constraints of electrical spatio-temporal additivity. However, an infinite number of weighted linear combinations may potentially account equally well for the observed data. As a consequence, the next step is to obtain simpler interpretations of the factors by performing a rotation procedure. Many different rotation procedures have been developed and used in the literature [63]. One of the most commonly used rotations is the Varimax [61], which provides the simplest data structure, and where the resulting factors are thought to be independent (orthogonal). The simplicity of Varimax rotation is characterized by the fact that the variance of the squared loadings is maximized. Thus, the Varimax identifies main factors whose relative contributions to the data tend to be large or small, not intermediate. The obvious consequence for its application to ERPs is that the resulting components tend to be either large or small at any time points; that is, this method minimizes the temporal overlap between different components. This specificity makes tPCA (and the Varimax rotation) particularly relevant to disentangle potentially overlapping ERP components (see also Fig. 2 here below). In addition, as already explained above, the extracted factors are thought to be independent one another, which may be useful when the goal is precisely to find out components specifically associated with experimental variables that are a priori assumed to be independent from each other (for instance testing for main effects in a factorial design). Note however that the independence assumption may constitute a limitation, particularly when exploring the underlying neural generators of the factors with source localization algorithms (see [3] for a thorough discussion). However, oblique rotations (e.g., Promax) can be used to circumvent the orthogonality assumption. Using Promax, each individual rotated factor is obtained regardless of its relationship with the other factors (see [63]). Being no longer strictly independent, the resulting rotated factors better meet the physiological assumptions required by most inverse solution algorithms.

The result of a tPCA is a set of factor loadings that correspond to the contribution of each new factor to the original variables (i.e., how much the temporal factor accounts for the voltage recorded at each time point). Thus the factor loadings can be seen as the elementary or basic waveforms, indicating segments in the ERPs during which a significant variability of amplitude is present. tPCA also provides a set of factor scores that correspond to the contribution of each factor to each independent variable (i.e., how much a temporal factor participate to the voltage observed for each original waveform). The factor scores indicate the nature of the variability that can then be analyzed by regular statistical tests. For instance, regrouping the scores by electrode reveals the topography of variability and sometimes, a subsequent spatial PCA on temporal factor scores is performed to reveal spatial components [71]. By contrast, regrouping the scores by condition reveals the experimental effects on variability. Depending on the research question, any combination of factor scores is feasible.

Finally, we should emphasize that although PCA is a data-driven analysis, its systematic application to ERPs requires some caution and expertise. In particular, the 
Fig. 2 Illustration of principal component analysis in a visual detection task with emotional picture targets. (a) Grand average ERPs for all 25 electrodes in response to targets (average collapsed across all three valence categories: unpleasant, pleasant and neutral, see [28]). The temporal window selected for the baseline to peak analysis is highlighted by the shaded rectangle. The horizontal scalp map shows the corresponding topography of this late positive peak. (b) Results of the tPCA (factor loadings) showing two distinct rotated factors (thin and thick waveforms corresponding to $\mathrm{P} 3 \mathrm{a}$ and $\mathrm{P} 3 \mathrm{~b}$ components, respectively), with distinct but partly overlapping time-courses. The topographical distribution of corresponding factor scores (unit-less values directly related to amplitude values) is shown on horizontal scalp maps
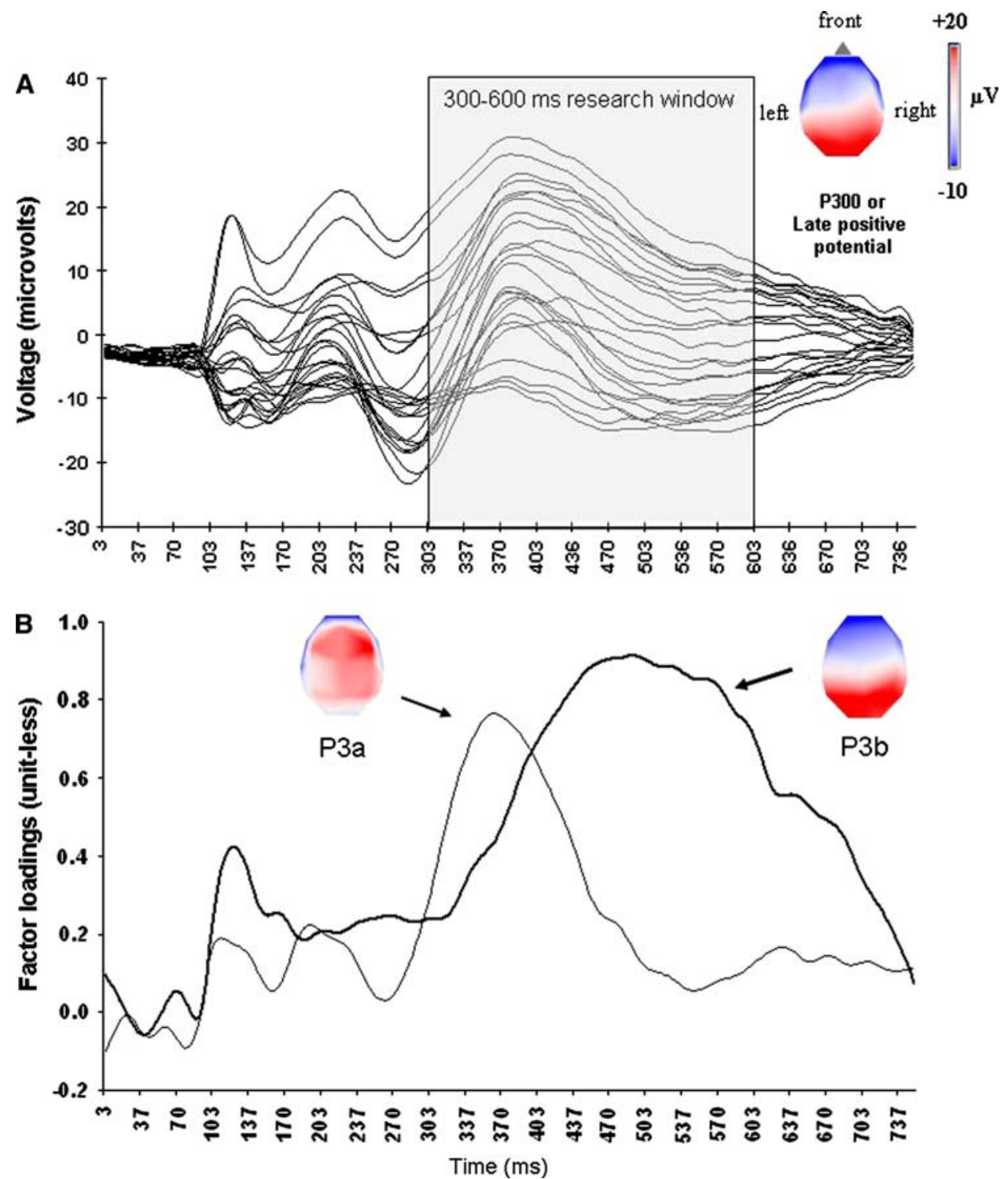

results may somewhat vary depending on the pre-selected matrix type, the decision to restrict the number of factors or not, and the rotation type (see [63-65]). PCA is also thought to be sensitive to latency jitters and misallocations of variance, although simulation studies have shown that these biases also influence baseline to peak analyses in conventional ERP analysis (and probably even more so). Moreover, these effects are actually negligible in comparison with the actual contributions of PCA [61, 62, 72].

\section{Using tPCA to Study Visual Emotion Perception}

Although the use of PCA is not restricted to any specific domain of cognition or emotion, PCA has been valuable to shed light on the spatio-temporal dynamics of visual emotion perception. For instance, given its ability to disentangle overlapping components, PCA was found to be particularly pertinent to explore long-latency (late) ERP components that are associated with emotion processing across a wide range of task conditions and stimulus categories [73]. Indeed, several studies have reported a reliable and sustained emotion-sensitive positive deflections in ERPs, with maximum amplitude over posterior recording sites, typically elicited between 300 and 1,000 ms after the onset of various emotional stimuli [74]. This component has been referred to as the late positive potential (LPP; [75, 76]), late positive complex (LPC; [77, 78]), or positive slow waves (PSW; [79]). This late positive component has also sometimes been interpreted as reflecting a P3b component [80-83], as classically recorded during an oddball paradigm [84]. But the relationship between this classic $\mathrm{P} 3 \mathrm{~b}$ and the late emotion-sensitive positive potential has generally remained unclear. 
A reliable finding is that the late positive potential is systematically augmented in amplitude in response to unpleasant or pleasant visual stimuli relative to neutral stimuli. This increase to emotional stimuli was usually interpreted as reflecting the processing of the arousal value of these visual stimuli, because this late positive component does not exhibit reliable effects of valence and correlates with measures of the autonomic nervous system associated with arousal (e.g., electrodermal activity, see [74]). However, this common observation (higher late positive potential for arousing relative to neutral visual stimuli) is usually obtained after having performed a conventional baseline to peak analysis for a fairly large temporal window [6], or after having averaged the amplitude values of many successive time points (e.g., average amplitude for successive $100 \mathrm{~ms}$ temporal windows starting $300 \mathrm{~ms}$ after stimulus onset and ending sometime $6 \mathrm{~s}$ after stimulus onset, [74]). Thus, in most studies (e.g., [74, $77,78,80,81])$, this late positive deflection in the ERPs is considered as a unitary long-lasting component. However, this assumption might be questioned, as it is unlikely in the context of active sensory or emotional processing that a single brain process would remain stable over such a long time range (e.g., for more than $500 \mathrm{~ms}$ ). Instead, it is possible that the late positive activity might result from several overlapping but distinct ERP components, which are difficult to tease apart when a conventional ERP analysis is used [14, 59]. In this context, a PCA decomposition may provide useful additional information, including the delineation of specific ERP components embedded within the same late positive potential.

Remarkably, already 20 years ago, Johnston et al. [85] used PCA to decompose ERPs evoked by visual emotion stimuli. These authors performed a tPCA (and used a Varimax rotation) allowing them to break down the late positive potential in what they labelled a P3 component, a P4 component, and a Slow Wave. These authors elegantly showed that the P3 and P4 were both sensitive to the emotional value of the pictures, while the Slow Wave was less specific, being both affected by the emotional content and the task. This ERP study was one of the first to suggest the added value of PCA in its ability to refine the timecourse of specific psychological variables (here with a focus on the emotional content and the task), each influencing the late positive potential of the ERPs, but with dissociable electrophysiological effects revealed by the PCA.

Consistent with the results of Johnston et al. [85], we also performed PCA of multi-channel ERP data (see [28]) to explore if the late positive activity evoked by visual emotion stimuli could be decomposed into distinct nonoverlapping components. In our study, we presented participants with unpleasant, pleasant and neutral pictures as targets in a standard oddball paradigm. Importantly, the mean arousal value was equated for the three categories. A coloured checkerboard served as frequent standard pictures, to which participants had not to respond (further details regarding the task, stimuli, EEG parameters and analyses can be found in Delplanque et al. [28]). The goal of this study was to test for any differential effect of picture valence on the early P3a versus the late P3b in visual ERPs, two components that may partly overlap in time (and topography), and that are difficult to separate from each other using a conventional baseline to peak analysis [14]. ERP components were extracted using a Varimax rotated tPCA. We found a striking dissociation when comparing ERP results obtained with the baseline to peak analysis and those obtained with the tPCA. In each case, we restricted the analysis to the same temporal window spanning 300$600 \mathrm{~ms}$ post-stimulus onset, which is commonly used in the ERP literature for selecting and measuring the P300 (see [86]).

Whereas the conventional baseline to peak analysis [6] revealed a single, uniform positive deflection peaking $380 \mathrm{~ms}$ post-stimulus onset (see Fig. 2a) consistent with either a non-specific P300 or an emotion-related LPP, our tPCA was able to separate two distinct sources of variability in the data during the exact same temporal window (Fig. 2b): one involved an early positive activity with a clear frontal scalp distribution (P3a), and the other involved a later positive activity with a parietal scalp distribution (P3b). Thus, the tPCA could accurately disentangle two partly overlapping but reliably distinct ERP components, while a conventional peak analysis only showed an undifferentiated large positive waveform (see also [85]).

In addition, statistical analyses (incorporating the normalization procedure of [87]) revealed no differential effect of stimulus valence on the amplitude values of the late positive activity measured using the baseline to peak method. By contrast, the same statistical analysis on data from the PCA indicated that, whereas the P3a amplitude was not altered by the valence of pictures, the P3b amplitude was substantially smaller for unpleasant compared to pleasant pictures (see [28]). This effect was replicated in a subsequent study using an emotional categorization task instead [29]. In addition, recent ERP studies based on PCA confirmed that the P3b was sensitive to both the valence and arousal of attended and unattended visual emotion stimuli, with these effects being modulated by the task at hand (e.g., when contrasting implicit versus explicit processing of the emotional content in stimuli; see [29, 30, $88]$ ). An effect of valence on the P300 was also suggested by Diedrich et al. [79] and by Conroy and Polich [86]. But these two ERP studies used baseline to peak analyses or computed average amplitude for successive time-points during temporal windows defined a priori, such that they 
could not draw strong conclusions about a genuine segregation of the underlying cognitive processes.

In our experiment reviewed above [28], the lack of significant valence effect on the late positive potential when using the baseline to peak analysis cannot be explained simply by a lack of power, but instead by the fact that this activity was likely to be subtended by a combination of two overlapping electric activities, each manifested by a distinct functional component (P3a and P3b). The baseline to peak analysis was blind to this dissociation. Thus, the use of PCA in ERP data analysis may help to refine the time-course of emotional effects on specific ERP components (such as the P300), which may arise simultaneously but influence separate cognitive processes. In the context of visual emotion perception [28-30], our PCA results offered new insights into the selective effects of core emotional dimensions such as valence or arousal on two different cognitive mechanisms such as the orienting of attention (P3a) and the updating of working memory (P3b).

Finally, we should point out that tPCA may help not only to break down late ERP effects (e.g., P300) into functionally distinct components during visual emotion processing, but may also be used to dissect earlier sensory stages in the visual ERPs to emotional stimuli (e.g., [89, 90]). For instance, PCA has been exploited to disentangle overlapping ERP components modulated by anxiety-related biases during emotion perception (including sub-components of the Contingent Negative Variation, see [91]); and to investigate effects of depression on visual emotion processing (including the P300 component, see [92]).

\section{Conclusions}

We have reviewed two distinct analysis techniques of multi-channel ERP data (microstate segmentation and PCA) which may be relevant to explore the time-course and exact electrophysiological correlates of complex mental processes such as emotion perception. These methods can provide unique additional insights that complement more conventional ERP analyses based on localized waveform peaks (for other alternative data-driven methods including Independent Component Analysis, see [3]). We first illustrated the advantages of microstate segmentation in the study of emotional attention [23, 32], where early topographic variations allowed us to identify neural activity in parietal areas that modulated spatial orienting towards emotional stimuli and provided top-down signals to enhance extrastriate responses to these emotional stimuli, but were not captured when using a conventional ERP analysis alone. Likewise, we illustrated the advantage of PCA in a study of visual emotion perception [28, 29], where temporally overlapping but functionally distinct neural responses could be separated into different factors, and thus reveal differential effects of the valence and arousal properties of visual target during the same time window (see also [29]), which were not detected when using a standard baseline to peak analysis.

To sum up, these two methods of analysis share in common the possibility to restrict the a priories in selecting a few channels or a few time-points that are thought (sometimes arbitrarily) to carry the relevant variance (or information) related to experimental variables. Although such analyses should not be performed on multi-channel ERP data sets without generating and testing specific hypotheses in the first place, we believe that additional and important insights about the time-course of emotion processing can be gained from these data analyses by allowing a holistic approach (i.e., considering all electrodes and time-points concurrently), and thus exploiting most usefully the complex information embedded within human EEG recordings.

Acknowledgements This work is supported by grants from the Swiss National Science Foundation (NCCR grant \# 51A240-104897; Swiss National Centre for Competence in Research in Affective Sciences).

\section{References}

1. Nunez PL. Electric fields of the brain: the neurophysics of EEG. London: Oxford University Press; 1981.

2. Nunez PL, Srinivasan R. Electric fields of the brain: the neurophysics of EEG. Oxford: Oxford University Press; 2005.

3. Makeig S, Debener S, Onton J, Delorme A. Mining event-related brain dynamics. Trends Cogn Sci 2004;8:204-10.

4. De Lucia M, Michel CM, Clarke S, Murray MM. Single-subject EEG analysis based on topographic information. Int $\mathrm{J}$ Bioelectromagn 2007;9:168-71.

5. Luck SJ. An introduction to the event-related potential technique. Cambridge MA: MIT Press; 2005.

6. Picton TW, Bentin S, Berg P, Donchin E, Hillyard SA, Johnson $\mathrm{R}$, et al. Guidelines for using human event-related potentials to study cognition: recording standards and publication criteria. Psychophysiology 2000;37:127-52.

7. Schupp HT, Flaisch T, Stockburger J, Junghofer M. Emotion and attention: event-related brain potential studies. Prog Brain Res 2006;156:31-51.

8. Lehmann D, Skrandies W. Reference-free identification of components of checkerboard-evoked multichannel potential fields. Electroencephalogr Clin Neurophysiol 1980;48:609-21.

9. Murray MM, Brunet D, Michel C. Topographic ERP analyses: a step-by-step tutorial review. Brain Topogr; this issue.

10. Skrandies W. The effect of stimulation frequency and retinal stimulus location on visual evoked potential topography. Brain Topogr 2007;20:15-20.

11. Michel CM, Murray MM, Lantz G, Gonzalez S, Spinelli L, Grave de Peralta R. EEG source imaging. Clin Neurophysiol 2004a; 115:2195-222.

12. Desmedt JE, Tomberg C, Noel P, Ozaki I. Beware of the average reference in brain mapping. Electroencephalogr Clin Neurophysiol Suppl 1990;41:22-7. 
13. Lehmann D. Principles of spatial analysis. In: Gevins AS, Remond D, editors. Handbook of electroencephalography and clinical neurophysiology. Methods of analysis of brain electrical and magnetic signals. Amsterdam: Elsevier; 1987. p. 309-54.

14. Spencer KM, Dien J, Donchin E. Spatiotemporal analysis of the late ERP responses to deviant stimuli. Psychophysiology 2001;38:343-58.

15. Geselowitz DB. The zero of potential. IEEE Eng Med Biol Mag 1998; 17:128-32.

16. Perrin F, Pernier J, Bertrand O, Echallier JF. Spherical splines for scalp potential and current density mapping. Electroencephalogr Clin Neurophysiol 1989;72:184-7.

17. Tucker DM, Liotti M, Potts GF, Russell GS, Posner MI. Spatiotemporal analysis of brain electrical fields. Hum Brain Mapp 1994;1:134-52.

18. Oostenveld R, Praamstra P. The five percent electrode system for high-resolution EEG and ERP measurements. Clin Neurophysiol 2001;112:713-9.

19. Michel CM, Seeck M, Landis T. Spatiotemporal dynamics of human cognition. News Physiol Sci 1999;14:206-14.

20. Michel CM, Thut G, Morand S, Khateb A, Pegna AJ, Grave de Peralta R, et al. Electric source imaging of human brain functions. Brain Res Brain Res Rev 2001;36:108-18.

21. Michel CM, Seeck M, Murray MM. The speed of visual cognition. Suppl Clin Neurophysiol 2004b;57:617-27.

22. Pourtois G, Dan ES, Grandjean D, Sander D, Vuilleumier P. Enhanced extrastriate visual response to bandpass spatial frequency filtered fearful faces: time course and topographic evoked-potentials mapping. Hum Brain Mapp 2005a;26:65-79.

23. Pourtois G, Vuilleumier P. Dynamics of emotional effects on spatial attention in the human visual cortex. Prog Brain Res 2006;156:67-91.

24. Brandeis D, Lehmann D. Event-related potentials of the brain and cognitive processes: approaches and applications. Neuropsychologia 1986;24:151-68.

25. Pascual-Marqui RD, Michel CM, Lehmann D. Segmentation of brain electrical activity into microstates: model estimation and validation. IEEE Trans Biomed Eng 1995;42:658-65.

26. Pourtois G, Grandjean D, Sander D, Vuilleumier P. Electrophysiological correlates of rapid spatial orienting towards fearful faces. Cereb Cortex 2004;14:619-33.

27. Pourtois G, Thut G, Grave de Peralta R, Michel C, Vuilleumier P. Two electrophysiological stages of spatial orienting towards fearful faces: early temporo-parietal activation preceding gain control in extrastriate visual cortex. Neuroimage 2005b;26: 149-63.

28. Delplanque S, Lavoie ME, Hot P, Silvert L, Sequeira H. Modulation of cognitive processing by emotional valence studied through event-related potentials in humans. Neurosci Lett 2004;356:1-4.

29. Delplanque S, Silvert L, Hot P, Rigoulot S, Sequeira H. Arousal and valence effects on event-related $\mathrm{P} 3 \mathrm{a}$ and $\mathrm{P} 3 \mathrm{~b}$ during emotional categorization. Int J Psychophysiol 2006;60:315-22.

30. Delplanque S, Silvert L, Hot P, Sequeira H. Event-related P3a and $\mathrm{P} 3 \mathrm{~b}$ in response to unpredictable emotional stimuli. Biol Psychol 2005;68:107-20.

31. Murray MM, Michel CM, Grave de Peralta R, Ortigue S, Brunet D, Gonzalez Andino S, et al. Rapid discrimination of visual and multisensory memories revealed by electrical neuroimaging. Neuroimage 2004;21:125-35.

32. Vuilleumier P. How brains beware: neural mechanisms of emotional attention. Trends Cogn Sci 2005;9:585-94.

33. Koenig T, Prichep L, Lehmann D, Sosa PV, Braeker E, Kleinlogel $\mathrm{H}$, et al. Millisecond by millisecond, year by year: normative EEG microstates and developmental stages. Neuroimage 2002;16:41-8.
34. Katayama H, Gianotti LRR, Isotani T, Faber PL, Sasada K, Kinoshita $\mathrm{T}$, et al. Classes of multichannel EEG microstates in light and deep hypnotic conditions. Brain Topogr 2007;20:7-14.

35. Vaughan HGJ. The neural origins of human event-related potentials. Ann N Y Acad Sci 1982;388:125-38.

36. Fender DH. Source localization of brain electrical activity. In: Gevins AS, Remond A, editors. Handbook of electroencephalography and clinical neurophysiology, vol. 1 methods of analysis of brain electrical and magnetic signals. Amsterdam: Elsevier; 1987. p. 355-99.

37. Srebro R. A bootstrap method to compare the shapes of two scalp fields. Electroencephalogr Clin Neurophysiol 1996;100:25-32.

38. Manly BF. Randomization, Monte Carlo methods in biology. London UK: Chapman \& Hall; 1991.

39. Kondakor I, Pascual-marqui RD, Michel CM, Lehmann D. Event-related potential map differences depend on the prestimulus microstates. J Med Eng Technol 1995;19:66-9.

40. Spierer L, Tardif E, Sperdin H, Murray MM, Clarke S. Learninginduced plasticity in auditory spatial representations revealed by electrical neuroimaging. J Neurosci 2007;27:5474-83.

41. He B, Lian J. High-resolution spatio-temporal functional neuroimaging of brain activity. Crit Rev Biomed Eng 2002;30:283-306.

42. Hämäläinen MS, Ilmoniemi RJ. Interpreting magnetic fields of the brain-minimum norm estimates. Med Biol Eng Comput 1994;32:35-42.

43. Pascual-Marqui RD, Michel CM, Lehmann D. Low resolution electromagnetic tomography: a new method for localizing electrical activity in the brain. Int J Psychophysiol 1994;18:49-65.

44. Grave de Peralta R, Gonzalez Andino S, Lantz G, Michel CM, Landis T. Noninvasive localization of electromagnetic epileptic activity. I. Method descriptions and simulations. Brain Topogr 2001;14:131-37.

45. Scherg M, von Cramon D. A new interpretation of the generators of BAEP waves I-V: results of a spatio-temporal dipole model. Electroencephalogr Clin Neurophysiol 1985;62:290-9.

46. MacLeod C, Mathews A, Tata P. Attentional bias in emotional disorders. J Abnorm Psychol 1986;95:15-20.

47. Mogg K, Bradley BP. Some methodological issues in assessing attentional biases for threatening faces in anxiety: a replication study using a modified version of the probe detection task. Behav Res Ther 1999;37:595-604.

48. Clark VP, Fan S, Hillyard SA. Identification of early visual evoked potential generators by retinotopic and topographic analyses. Hum Brain Mapp 1995;2:170-87.

49. Egeth HE, Yantis S. Visual attention: control, representation, and time course. Ann Rev Psychol 1997;48:269-97.

50. Vogel EK, Luck SJ. The visual N1 component as an index of a discrimination process. Psychophysiology 2000;37:190-203.

51. Hillyard SA, Vogel EK, Luck SJ. Sensory gain control (amplification) as a mechanism of selective attention: electrophysiological and neuroimaging evidence. Philos Trans R Soc Lond B Biol Sci 1998;353:1257-70.

52. Kastner S, Ungerleider LG. Mechanisms of visual attention in the human cortex. Annu Rev Neurosci 2000;23:315-41.

53. Foxe JJ, Simpson GV. Flow of activation from V1 to frontal cortex in humans. A framework for defining "early" visual processing. Exp Brain Res 2002;142:139-50.

54. Pegna AJ, Khateb A, Spinelli L, Seeck M, Landis T, Michel CM. Unraveling the cerebral dynamics of mental imagery. Hum Brain Mapp 1997;5:410-21.

55. Murray MM, Camen C, Gonzalez Andino SL, Bovet P, Clarke S. Rapid brain discrimination of sounds of objects. J Neurosci 2006;26:1293-302.

56. Arzy S, Mohr C, Michel CM, Blanke O. Duration and not strength of activation in temporo-parietal cortex positively correlates with schizotypy. Neuroimage 2007;35:326-33. 
57. Scherer KR. Appraisal considered as a process of multilevel sequential checking. In: Scherer KR, Schorr A, Johnstone T, editors. Appraisal processes in emotion: theory, methods, research. New York and Oxford: Oxford University Press; 2001.

58. Lefèvre J, Obozinski G, Baillet S. Imaging brain activation streams from optical flow computation on 2-Riemannian manifolds. Inf Process Med Imag 2007;20:470-81.

59. Spencer KM, Dien J, Donchin E. A componential analysis of the ERP elicited by novel events using a dense electrode array. Psychophysiology 1999;36:409-14.

60. Skrandies W. Data reduction of multichannel fields: global field power and principal component analysis. Brain Topogr 1989;2:73-80.

61. Chapman RM, McCrary JW. EP component identification and measurement by principal components analysis. Brain Cogn 1995;27:288-310.

62. Van Boxtel GJM. Computational and statistical methods for analyzing event-related potential data. Behav Res Meth Instrum Comp 1998;30:87-102.

63. Dien J, Beal DJ, Berg P. Optimizing principal components analysis of event-related potentials: matrix type, factor loading weighting, extraction, and rotations. Clin Neurophysiol 2005; 116:1808-25

64. Kayser J, Tenke CE. Optimizing PCA methodology for ERP component identification and measurement: theoretical rationale and empirical evaluation. Clin Neurophysiol 2003;114:2307-25.

65. Kayser J, Tenke CE. Trusting in or breaking with convention: towards a renaissance of principal components analysis in electrophysiology. Clin Neurophysiol 2005;116:1747-53.

66. Kayser J, Tenke CE. Principal components analysis of Laplacian waveforms as a generic method for identifying ERP generator patterns: I. Evaluation with auditory oddball tasks. Clin Neurophysiol 2006a; 117:348-68.

67. Kayser J, Tenke CE. Principal components analysis of Laplacian waveforms as a generic method for identifying ERP generator patterns: II. Adequacy of low-density estimates. Clin Neurophysiol 2006b;117:369-80.

68. Lagerlund TD, Sharbrough FW, Busacker NE. Use of principal component analysis in the frequency domain for mapping electroencephalographic activities: comparison with phase-encoded Fourier spectral analysis. Brain Topogr 2004;17:73-84.

69. Tiejun L, Dezhong Y. Removal of the ocular artifacts from EEG data using a cascaded spatio-temporal processing. Comp Meth Prog Biomed 2006;95-103.

70. Kayser J, Fong R, Tenke CE, Bruder GE. Event-related brain potentials during auditory and visual word recognition memory tasks. Brain Res Cogn Brain Res 2003;16:11-25.

71. Carretie L, Hinojosa JA, Mercado F, Tapia M. Cortical response to subjectively unconscious danger. Neuroimage 2005;24:615-23.

72. Beauducel A, Debener S. Misallocation of variance in eventrelated potentials: simulation studies on the effects of test power, topography, and baseline-to-peak versus principal component quantifications. J Neurosci Meth 2003;124:103-12.

73. Vuilleumier P, Pourtois G. Distributed and interactive brain mechanisms during emotion face perception: evidence from functional neuroimaging. Neuropsychologia 2007;45:174-94.

74. Cuthbert BN, Schupp HT, Bradley MM, Birbaumer N, Lang PJ. Brain potentials in affective picture processing: covariation with autonomic arousal and affective report. Biol Psychol 2000; 52:95-111.
75. Bensafi M, Pierson A, Rouby C, Farget V, Bertrand B, Vigouroux $\mathrm{M}$, et al. Modulation of visual event-related potentials by emotional olfactory stimuli. Neurophysiol Clin 2002;32:335-42.

76. Schupp HT, Cuthbert BN, Bradley MM, Cacioppo JT, Ito T, Lang PJ. Affective picture processing: the late positive potential is modulated by motivational relevance. Psychophysiology 2000;37:257-61.

77. Ito TA, Larsen JT, Smith NK, Cacioppo JT. Negative information weighs more heavily on the brain: the negativity bias in evaluative categorizations. J Person Soc Psychol 1998;75:887-900.

78. Ito TA, Cacioppo JT. Electrophysiological evidence of implicit and explicit categorization processes. J Exp Soc Psychol 2000;36:660-76.

79. Diedrich O, Naumann E, Maier S, Becker G, Bartussek D. A frontal positive slow wave in the ERP associated with emotional slides. J Psychophysiol 1997;11:71-84.

80. Naumann E, Bartussek D, Diedrich O, Laufer ME. Assessing cognitive and affective information processing functions of the brain by means of the late positive complex of the evnet-related potential. J Psychophysiol 1992;11:285-98.

81. Naumann E, Maier S, Diedrich O, Becker G, Bartussek D. Structural, semantic, and emotion-focussed processing of neutral and negative nouns: event-related potential correlates. J Psychophysiol 1997;11:158-72.

82. Cuthbert BN, Schupp HT, Bradley M, McManis M, Lang PJ. Probing affective pictures: attended startle and tone probes. Psychophysiology 1998;35:344-47.

83. De Pascalis V, Strippoli E, Riccardi P, Vergari F. Personality, event-related potential (ERP) and heart rate (HR) in emotional word processing. Pers Individ Dif 2004;36:873-91.

84. Sutton S, Braren M, Zubin J, John ER. Evoked-potential correlates of stimulus uncertainty. Science 1965;150:1187-8.

85. Johnston VS, Miller DR, Burleson MH. Multiple P3s to emotional stimuli and their theoretical significance. Psychophysiology 1986;23:684-94.

86. Conroy MA, Polich J. Affective valence and P300 when stimulus arousal level is controlled. Cogn Emot 2007;21:891-901.

87. McCarthy G, Wood CC. Scalp distributions of event-related potentials: an ambiguity associated with analysis of variance models. Electroencephalogr Clin Neurophysiol 1985;62:203-8.

88. Hot P, Saito Y, Mandai O, Kobayashi T, Sequeira H. An ERP investigation of emotional processing in European and Japanese individuals. Brain Res 2006;1122:171-8.

89. Carretie L, Hinojosa JA, Lopez-Martin S, Tapia M. An electrophysiological study on the interaction between emotional content and spatial frequency of visual stimuli. Neuropsychologia 2007;45:1187-95.

90. Rigoulot S, Delplanque S, Despretz P, Defoort-Dhellemmes S, Honoré J, Sequeira H. Peripherally presented emotional scenes: a spatiotemporal analysis of early ERP responses. Brain Topogr; this issue.

91. Carretie L, Mercado F, Hinojosa JA, Martin-Loeches M, Sotillo M. Valence-related vigilance biases in anxiety studied through event-related potentials. J Affect Disord 2004;78:119-30.

92. Kayser J, Bruder GE, Tenke CE, Stewart JE, Quitkin FM. Eventrelated potentials (ERPs) to hemifield presentations of emotional stimuli: differences between depressed patients and healthy adults in P3 amplitude and asymmetry. Int $\mathrm{J}$ Psychophysiol 2000;36:211-36. 